\title{
Algebraic QFT and the area law for entropy of localized quantum matter
}

\author{
Bert Schroer* \\ CBPF, Rua Dr. Xavier Sigaud 150 \\ 22290-180 Rio de Janeiro, Brazil \\ and Institut fuer Theoretische Physik der FU Berlin, Germany
}

\begin{abstract}
The algebraic approach to QFT, which for several decades has enriched QFT with structural theorems, has recently shown its utility in various constructions of actual interest. After some introductory remarkss which illustrate this point, the main content of the paper addresses the issue of algebraic holography. The example of lightfront holography is presented in some detail, and its use in studying thermal aspects of localization is explained. The vacuum polarization at the edge of the causal horizon of a wedge-localized algebra is identified as the cause of an area proportionality of localization-entropy of localized quantum matter. Finally the adaptation of the method to the analogous case of black holes with bifurcated Killing horizons is sketched.
\end{abstract}

Fifth International Conference on Mathematical Methods in Physics

24 - 28, April 2006

Rio de Janeiro, Brazil

\footnotetext{
* Speaker.
} 


\section{Motivations for an alternative approach to QFT}

Looking at the present particle physics scene one may get the impression that besides string theory and loop quantum gravity there are no other worthwhile fundamental alternatives for a post standard model particle physics.

There exist however of $a$ third way whose pursuit neither requires to wait for the moment when new experimental data resolve a theoretical impasse (in the present situation the problems around the Higgs particle and the fate of supersymmetry), nor does it involve the risks of getting lost in the blue yonder of highly speculative ideas.

This third way consists in expanding the conceptual basis of QFT in order to explore other directions. Although one has a good knowledge of the underlying principles and of its conceptual and mathematical setting, QFT in contrast to QM remains a hugely unfinished project. The rapid progress particle physics enjoyed during several decades of the last century has left huge gaps of knowledge with many important problems having been left on the wayside. In fact it is surprising that by only knowing a perturbative approach, whose conceptual status is still shrouded in doubts as a result of arguments pointing to the divergence of the perturbative series, one nevertheless was able to find the standard model whose descriptive precision (after observationally fixing its many free parameters) leaves nearly nothing to be desired. This third way was always available, but in good times of the past, when ideas supported by the standard Lagrangian computational methods led to a lot of progress, there was no strong motivation to explore the intrinsic logic of QFT and to look for alternative directions. Our knowledge has mainly been obtained by canonical or functional integral quantization of classical objects, i.e. by a parallelism to a world of classical field theory, or in the words of one of the the protagonists of field quantization Pascual Jordan, with the help of "classical crutches" [1].

The research of some dedicated individuals over several decades gradually led to an autonomous setting of QFT which is generally referred to as algebraic QFT (AQFT) or local quantum physics (LQP) [2]. It was quite successful in obtaining various observable properties from a few fundamental principles and to get a conceptually consistent finite formulation of perturbative renormalization [3]. But on the whole its main role was more that of a critical corrective than of a catalyzer for new discoveries. The present crisis in particle physics, in particular the stagnation in attempts in understanding the problems which the standard model (SM) left behind after its impressive initial success, ask for a fresh look at an alternative conceptual setting of QFT. It seems that the formulation of QFT which was instrumental in its discovery, namely that in terms of Lagrangians and gauge symmetries, is not well suited to address the many new problems which arose from its perturbative exploration.

Since there are growing doubts about the existence of an autonomous "gauge principle" in QFT (the use of gauge invariance as a technical tool in perturbative renormalization remains unaffected), it is important to know whether the locality principle in conjunction with renormalizability requires interacting massive vectormesons to be accompanied by scalar particles. Clearly the Lagrangian setting is not a good point of departure for investigating fundamental problems of this kind; the best one can do in the standard setting is to start with coupled massive vectormesons and verify that without introducing additional scalar particles (the Higgs field, but without its characteristic vacuum expectation) the BRST gauge formalism would not work i.e. the cohomological descend 
to a ghostfree covariant Hilbert space description would fail [ [ a formalism which relies on unphysical and nonobservable concepts as ghosts is not trustworthy; If we want to heed Heisenberg's message about the importance of staying with observables in fundamental arguments of QT, we are forced to look at an alternative ghostfree implementation of interactions for particles of $\operatorname{spin} \geq 1$.

The Wigner representation theoretical approach for the Poincaré group turned out to be a good starting point for a ghostfree description. Among the positive energy representations in $d=3+1$ there are two zero mass families whose associated QFT cannot be described in terms of pointlike covariant fields. One family is associated with the so-called continuous spin representations which are characterized by a continuous-valued invariant of the Wigner "little group" of a lightray; the second is the finite helicity family to which the photon and the (still elusive) graviton belong. To be more precise, in the finite helicity case it is not the field strength (electromagnetic field strength, the linearized Riemann tensor field strength,...) which forces one to go beyond point-like localized fields, but rather the "potentials" of these field strengths. One knows from the experience with QED that one needs the potentials for the formulation of interactions and there are even intrinsic theoretical reasons for their introduction [5]. As a consequence of a well-known representation theoretical no-go theorem concerning covariant point-like localized vectorpotentials in the physical representation space, one is forced to relax the localization aspect. One finds that the best one can do in a Wigner-Fock space is to construct covariant fields $A_{\mu}(x, e)$ which are localized on a semi-infinite string $x+\mathbb{R}_{+} e$ (where $e$ is a spacelike unit vector) [5]. Their commutator is "stringlocal" i.e. vanishes only if the two strings do not enter their respective causal influence regions. These string-like localized potentials transform covariant under the unitary Wigner representation of the Poincaré group. This field fluctuates simultaneously in $x$ and in the space-like direction $e$, i.e. $e$ is not an additional parameter with a fixed value, but rather behaves like a localization point in a $\mathrm{d}=2+1 \mathrm{de}$ Sitter spacetime. Apart from its participation in the Poincare transformations, the $e$ are reminiscent of the axial gauge. But whereas the interpretation of $e$ as a gauge causes nasty infrared problems, taking into account its singular fluctuating nature (distribution in $e$ ) leads to informations of how to treat this dependence properly. Since part of the vacuum fluctuations are transferred to $e$, the short distance dimension in $x$ is reduced to 1, i.e. the string-localized potential has the same short distance behavior as the point-like potential in the BRST ghost setting. This idea of constructing short-distance improved string-localized free fields works also in the case of massive vectormesons when there is no representation theoretical reason for their introduction. If it would be possible to formulate a renormalized perturbation theory for string-like localized massive vectormesons, one would have a conceptually clear starting point to clarify the possible necessity for additional physical degrees of freedom of the Higgs type. The $e$-independence would then play the role of the gauge invariance in the BRST formalism; but since $e$-independence means locality, the gauge principle would be replaced by the more fundamental locality principle and the discussion about the necessity of Higgs degrees of freedom would be shifted to a more conceptual level than that obtained from the setting of Lagrangians and functional integrals. More remarks on the use of string-localization can be found in the contribution by J. Mund to this conference. In the present context it only served to illustrate that there are important problems for which the representation theoretic or algebraic setting opens new avenues which are not available with Lagrangians and functional integrals. 
The most impressive illustration of the power of alternative conceptual ideas originating from AQFT comes from the recent solution of the old problem of how to incorporate quantum matter into general relativity, more precisely how to extend Einstein's principle of local covariance to QFT in curved space time (CST). Since the obstruction against local diffeomorphism covariance comes from the inherent global aspects of quantum states, the important step was to find a way to separate the construction of the operator-algebraic aspects from that of states. Clearly the standard functional integral formalism which aims at correlation functions, i.e. at expectation values of operators in a globally invariant vacuum states, is not a good starting point for theories in which local diffeomorphisms play a role. The search for a better formulation started with the problem of defining the correct locally covariant Wick-products of free fields in curved spacetime, in particular with the search for a quantum energy-momentum tensor which only depends on the spacetime in the neighborhood of its point of definition [7]. The standard "split point" method of taking the product of free fields at different points and subtracting its expectation in a suitable state before taking the limit of coalescing points did not work since every state introduces an undesired dependence on global properties of spacetime.

After more than one decade of conceptional innovative mathematical work, a highly satisfactory framework was finally found. Its solution requires to take a new view about the basics of QFT [6]. Whereas in the algebraic setting of QFT as originally proposed by Haag and elaborated over several decades by many authors [2] the starting point was a system of operator algebras indexed by subregions of a fixed spacetime, the quantum implementation of local covariance requires to do this simultaneously on all physically admissible spacetimes. This is because Einstein's local covariance principle demands that the physics of two admissible isometric diffeomorphic patches on two different globally hyperbolic Lorentz manifolds is isomorphic; by only allowing operations restricted to those patches one cannot decide in which world one is living. In this way QFT in curved spacetime becomes a functorial concept between a suitable category of Lorentz manifolds and a category of suitably defined operator algebras. As it should be, the old setting of AQFT in Minkowski spacetime is a special case; in mathematical terms, the Haag-Kastler axioms re-emerge from the new functorial setting.

One would expect that such an important discovery should rapidly become part of the general scientific discourse, but in this case this did not (yet?) happen. Perhaps it is one aspect of the present crisis that the euclidean setting which is important for making mathematical sense of functional integral has gained prominence among physicists inspite of its limited physical applicability; many particle physicists who are familiar with euclidean methods (and use them even outside their range of applicability in theories involving gravity) have only a very rudimentary knowledge of those operator algebraic structures as they are used in the formulation of the new local covariance principle. But there can be no doubt that this new algebraic setting which implements the local covariance principle is the most important paradigmatic change within QFT after the theoretical discovery of black hole radiation. In [10] the reader finds interesting remarks how to get from local covariance to diffeomorphism invariance (background independence) which is believed to be a characteristic property of quantum gravity. These observations raise questions about the validity of claim that gravity is incompatible with the principles of quantum theory, which is the main motivational argument for string- and loop- gravity.

In the next section I will present some aspects of recent work on a related subject namely 
algebraic holography and localization-entropy which may be relevant for black hole physics.

\section{Remarks on lightfront holography}

In the algebraic setting of QFT the concept of holographic projection on null-surfaces means the replacement of the system of subalgebras, which are associated with subregions in the bulk, by a new system of subalgebras which are indexed with respect to subregions of its causal horizon. Hence If one, following Leibniz, considers spacetime as an ordering device for (quantum) matter, holography amounts to a change of the ordering device while maintaining the material substrate.

The best studied case is the lightfront holography [8] which starts from the geometric fact that the light front $L F$ is the linear extension of the upper horizon of a fixed wedge $W$ region (all regions are open). The $x_{0}-x_{3}$ wedge is defined as the region $W=\left\{\left|x_{0}\right|<x_{3}\right\}$ with the transverse spatial coordinates remaining unrestricted. The aim is to construct a system of subalgebras indexed by subregions on its (always upper) boundary $\partial W$. The concept which mediates between the bulk in $W$ and its holographic projection on $\partial W$ is the causal shadow property of the algebraic approach. It states that an operator algebra associated with a region $\mathscr{O}$ is equal to that associated with the (generally larger) causally completed region $\mathscr{O}^{\prime \prime}$ (taking twice the causal disjoint). Since $W$ is the (past) causal shadow of $\partial W$ (every signal which passes through $\partial W$ must have traversed $W$ ), one identifies the algebra belonging to the characteristic surface $\partial W$ with that of $W$ i.e. $\mathscr{A}(\partial W) \equiv$ $\mathscr{A}(W)$. The linear extension of $\partial W$ is a lightfront $L F$ with $\mathscr{A}(L F)=\mathscr{A}(M)$ since its causal shadow is the full algebra of Minkowski spacetime. The only smaller regions in $L F$ which cast an ambient causal shadow are horizons of wedges i.e. of those wedges which have their horizon on $L F$. The aim of hologaphy on $L F$ is to construct a coherent system of subalgebras $\mathscr{A}(\mathscr{O}) \subset \mathscr{A}(\partial W) \subset \mathscr{A}(L F)$ where $\mathscr{O} \subset \partial W \subset L F$ are regions which do not cast causal shadows; the important tool is the formation of intersections of algebras, starting from the wedge algebras in the bulk.

The symmetry group $\mathscr{G}$ of $L F$ is a 7-parametrig subgroup of the 10-parametric Poincaré group, consisting of lightray translations, dilations (the $L F$ projections of the $\mathrm{W}$-preserving boost), linear transformations of the coordinate directions in the transverse space $\mathbb{R}^{2} \subset L F$ (resulting from the $L F$ projection of the 3-parameter Wigner little group which leaves the lightray invariant) as well as transverse translations. The local subalgebras $\mathscr{A}(\mathscr{O}), \mathscr{O} \subset L F$ of the holographic projection on $L F$ are obtained by successive steps involving intersections of wedge algebras obtained from $W$ by the application of $\mathscr{G}$.

Let us briefly look how this is done. Let $W$ be the $x_{0}-x_{3}$ wedge which is invariant under $x_{0}-x_{3}$ boosts. Consider a family of wedges $W_{a}$ which are obtained by sliding the $W$ along the $x_{+}=x_{0}+x_{3}$ lightray by a lightlike distance $a>0$ into itself. The region on $L F$ consisting of those points on $\partial W_{a}$ which are spacelike to the interior of $W_{b}$ for $b>a$ is denoted by $\partial W_{a, b}$ and consists of points $x_{0}=x_{3}, x_{+} \in(a, b)$ with an unlimited transverse part $x_{\perp} \in R^{2}$. These regions are slabs on $L F$ with the full $\mathbb{R}^{2}$ transverse extension. To get to regions of finite transverse size one first transforms this slabs by the action of the 2-parametric subgroup $\mathscr{G}_{2}$ of $\mathscr{G}$ which is the restriction to $L F$ of the two "translations" in the Wigner little group (the subgroup fixing the lightray in $L F$ ) and afterwards intersects the transformed slab with $\partial W_{a, b}$

$$
\partial W_{a, b} \cap g\left(\partial W_{a, b}\right), g \in \mathscr{G}_{2}
$$


By forming intersections and unions one can get to finite convex regions $\mathscr{O}$ of a more general shape. An alternative method for obtaining holographically projected compactly localized subalgebras $\mathscr{A}(\mathscr{O}), \mathscr{O} \subset L F$, which does not make use of transverse symmetries, consists in intersecting $\mathscr{A}\left(\partial W_{a}\right)$ with suitable algebras in the bulk which are localized in a tubular neighborhood of $\mathscr{O}[9]$.

The main problem is now to show the nontriviality of the algebras associated to those regions. Since any region on $\partial W$ which does not extend to infinity in the $x_{+}$lightray direction also does not cast any causal shadow, we cannot base the nontriviality of algebras $A\left(\partial W_{a, b}\right)$ on the causal shadow property. If this intersected algebra would be trivial (i.e. consist of multiples of the identity), the idea of holographic projections would be useless.

It has been customary to add structural properties concerning intersections to the list of algebraic requirements if they can be established in the algebraic reformulation of free fields. Since one knows very little about the existence of interacting QFT, this is a reasonable procedure. It is interesting to note that very recently nontriviality proofs for algebraic intersection of wedge algebras of special interacting models have been found which led to the first existence proofs of the associated 2-dim. QFT models [12]. These wedge algebras have generators with rather simple vacuum polarization properties [11].

Returning to free fields it is well-known that a nontrivial system of localized operator algebras $\mathscr{A}(\mathscr{O}), \mathscr{O} \subset L F$ exists [13]; in fact these algebras on $L F$ have simple pointlike generators $A_{L F}$ which are identical to the fields which appeared in the "lightcone quantization" (or $p \rightarrow \infty$ frame) formalism

$$
\begin{aligned}
& A_{L F}\left(x_{+}, x_{\perp}\right)=\frac{1}{(2 \pi)^{3 / 2}} \int\left(e^{i\left(p_{-}(\theta) x_{+}\right.} a^{*}\left(\theta, p_{\perp}\right) \frac{d \theta}{2} d p_{\perp}+h . c .\right), p_{-}(\theta)=m e^{\theta} \\
& \left\langle\partial_{x_{+}} A_{L F}\left(x_{+}, x_{\perp}\right) \partial_{x_{+}} A_{L F}\left(x_{+}^{\prime}, x_{\perp}^{\prime}\right)\right\rangle \simeq \frac{1}{\left(x_{+}-x_{+}^{\prime}+i \varepsilon\right)^{2}} \cdot \delta\left(x_{\perp}-x_{\perp}^{\prime}\right) \\
& {\left[\partial_{x_{+}} A_{L F}\left(x_{+}, x_{\perp}\right), \partial_{x_{+}} A_{L F}\left(x_{+}^{\prime}, x_{\perp}^{\prime}\right)\right] \simeq \delta^{\prime}\left(x_{+}-x_{+}^{\prime}\right) \delta\left(x_{+}-x_{\perp}^{\prime}\right)}
\end{aligned}
$$

Here all unimportant constants are left out and the derivative is used in order to avoid the technicalities related to infrared aspects of logarithmic zero mass correlations. The $A_{L F}$ field is different from the original free field; the only physical purpose of this auxiliary field is to generate the system of local operator algebras on $L F$. The operator algebras $\mathscr{A}(\mathscr{O}) \subset \mathscr{A}(L F)$ are constructed by first smearing $A_{L F}$ with $\mathscr{O}$-localized testfunctions on $L F$ and then passing to generating bounded operators of $\mathscr{A}(\mathscr{O})$ using the Weyl exponentiation [13]; the construction is completely analogous to the way one passes from free fields to their associated spacetime localized operator algebras with the only difference being that the Minkowski spacetime is replaced by $L F$.

The factorization of the $A_{L F}$ correlation into a lightlike and a transverse part reveals two important properties. The transverse quantum mechanical delta function shows that there are no transverse vacuum fluctuation. All vacuum fluctuations have been compressed into the lightlike direction with the result of an increase of lightlike symmetry. Whereas the lightray translation was part of the original Poincaré symmetry and the dilation on $L F$ results from the $W$-preserving boost, the chiral rotation (which together with the translation and the dilation generate the Moebius group) is a new symmetry.

These observations pose the question whether absence of transverse vacuum fluctuations and the appearance of chiral symmetries in the lightray direction are general properties of holographic 
lightfront projection. The appearance of stronger short-distance singularity in the presence of interactions prevents a direct definition of $A_{L F}$ fields via restriction of bulk fields to $L F$, and one is forced to follow the afore-mentioned more abstract algebraic path. One of the "magic" aspects of the algebraic approach is that certain positions of operator algebras relative to each other (acting in the same Hilbert space) may lead to additional geometric symmetries. For the case at hand it is the fact that the algebras $\mathscr{A}\left(\partial W_{a}\right), \mathscr{A}\left(\partial W_{b}\right)$ and their relative commutant $\mathscr{A}\left(\partial W_{a, b}\right)$ define a standard modular inclusion and that this property characterizes chiral theories [14]. The existence of a vacuum-preserving unitary implementation of the rotation on the compactified lightray in the general setting is a consequence of the algebraic properties of the $L F$ holography.

The chiral part of the $A_{L F}$ correlation function admits a much larger unitarily implemented symmetry group namely the diffeomorphism group of the circle. The transformations beyond the Moebius group do not leave the vacuum invariant and hence are not Wigner symmetries. It is not clear whether this Diff $\left(\mathrm{S}^{1}\right)$ also exists in the interacting case. The standard argument for its validity is based on the existence of an energy-momentum tensor and unlike chiral theories which originate from two-dimensional conformal theories, this argument is not available for chiral theories associated with holographic projections. As the vacuum preserving chiral rotation results from algebraic properties of the holographic projection, it is conceivable that one finds also operator-algebra properties which lead to the higher diffeomorphism symmetries. An extension to local diffeomorphisms would be very much in the spirit of the new setting of QFT based on the implementation of local covariance [6]. Note that the unitary implementers of symmetries which originate from holography are also well-defind in the bulk theory since they act in the same Hilbert space; however their action on the bulk is not geometric, i.e. they are not symmetries in the sense of the Noether theorem of the bulk.

The important aspect of holography is the absence of transverse vacuum polarization. This is a consequence of the fact that the lightlike translation, different from space- or time- like translations, fulfills two properties simultaneously: its generator has positive spectrum and the lightlike translation fulfills a cluster property for large lightlike distances. Let $\mathscr{O}_{i}, i=1,2$ be two regions on $L F$ whose transverse projections do not overlap i.e. $\left(\mathscr{O}_{1}\right)_{\perp} \cap\left(\mathscr{O}_{2}\right)_{\perp}=\emptyset$, then the following factorization holds ${ }^{1}$

$$
\langle\Omega|A B| \Omega\rangle=\langle\Omega|A| \Omega\rangle\langle\Omega|\mathscr{B}| \Omega\rangle, A \in \mathscr{A}\left(\mathscr{O}_{1}\right), B \in \mathscr{A}\left(\mathscr{O}_{2}\right)
$$

Note that since the regions are open, this factorization even holds in case the two regions touch on their boundaries. In view of the fact that this never happens in the bulk, this is quite surprising; in fact the simplification in terms of vacuum fluctuations is the main raison d'être for holography in the sense of the present work. We remind the reader that even for a finite spacelike separation of two localization regions in the bulk there can be no factorization on the vacuum vector $\Omega$; in order to construct state vectors which lead to tensor-factorization in the bulk one has to invoke the split property which is known to break down in the case that the two regions touch [2].

It is precisely this absence of transverse vacuum polarization in the holographic projection which simplifies the description of thermal manifestations of localization. It compresses the vacuum fluctuations into the lightlike direction and the relevant Hamiltonian for the thermal properties

\footnotetext{
${ }^{1}$ Locality in both directions shows that the lightlike translates $\langle\Omega|A U(a) B| \Omega\rangle$ are boundary values of entire functions and the cluster property together with Liouville's theorem gives the factorization [13].
} 
of localization is the lightlike dilation generator which leaves $\partial W$ invariant and whose properties can be studied by methods of chiral QFT. The transverse decoupling implies transverse additivity of extensive quantities and hence imposes an area proportionality on entropy and energy caused by the vacuum fluctuations on the horizon in the lightray direction at the $x_{+}=0$ boundary of $\partial W$. Since one expects this area density to diverge in the limit of a sharp $x_{+}=0$ boundary, the strategy will be to introduce an "attenuation length" $\varepsilon$ for the vacuum fluctuations and then study the limiting behavior for $\varepsilon \rightarrow 0$.

The computation of the leading $\varepsilon$-divergence is based on a theorem [8] which states that a chiral system in a vacuum state $\Omega$, localized on a halfline $\mathbb{R}_{+}$, can be unitarily mapped to the full chiral algebra on $\mathbb{R}$ in a KMS thermal state $\Omega_{2 \pi}$ at temperature $2 \pi$

$$
\left(\mathscr{A}\left(\mathbb{R}_{+}\right), \Omega\right) \simeq\left(\mathscr{A}(\mathbb{R}), \Omega_{2 \pi}\right)
$$

with the unitary equivalence being given in terms of a conformal map which intertwines the dilation of the $\mathbb{R}_{+}$-restricted system with the translation of the unrestricted KMS thermal system. This "inverse Unruh effect" for chiral theories (i.e. a heat bath system is interpreted as a spacetimerestricted vacuum system) is the key to the calculation of localization-entropy. Using the standard wisdom of statistical mechanics we would conjecture that the entropy diverges proportional to the length as $l \cdot s_{2 \pi}$ where $s_{2 \pi}$ is the density per length. The conformal equivalence map intertwines the translation of the heat bath system with the dilation of the localized system so that the size $l$ is transformed into $\varepsilon$ according to $\varepsilon=e^{-l}$ and the area density of the equivalent dilational system on the half-space behaves as

$$
s_{\text {area }}=|\ln \varepsilon| s_{2 \pi}+\text { finite }, \varepsilon \rightarrow 0
$$

The correctness of this idea can be checked by approximating the linear system by a sequence of finite systems using an "invariant" box approximation [8] in which the divergent partition function of the translative system is approximated by a sequence of rotational system in the limit of infinite temperature (interpreted as an infinite radius whose size is related to $l$ ) associated with the partition function of the Virasoro generator $L_{0}$. The calculation can be completed by the use of the chiral temperature duality for the partition function of $\hat{L}_{0}=L_{0}-\frac{c}{24}$ where the $c$ is the Virasoro constant. It is precisely this shift in $L_{0}$ which gives the divergent $l$ factor and identifies the constant according to $s_{2 \pi}=\frac{c}{12}[8]$.

As mentioned before chiral theories which originate from the chiral decomposition of conformal two-dimensional theories come with an energy momentum tensor whose strength defines the Virasoro constant $c$, but this is not necessarily the case for Moebius covariant chiral theories which arise in holographic lightfront projection. The fact that the vacuum shift to $\hat{L}_{0}$ which is necessary for the formulation of temperature duality also leads to the expected large $l$ behavior may be taken as an indication that the Virasoro structure continues to hold for chiral theories which are associated with holographic projections. At the same time it gives an apparently new thermal interpretation to the Virasoro constant $c$ in terms of properties of a lightlike thermal system at KMS temperature $2 \pi$. This "inverse Unruh effect" in combination with the simplification from the holographic projection makes localization-entropy a really useful concept.

It is an interesting question to what extend such entropy considerations apply to other nullsurfaces in Minkowski spacetime as e.g. the upper horizon of double cones which are conformally 
equivalent to wedges. Since the thermal phenomenon under consideration is caused by vacuum fluctuations near the boundary, one expects that the localization-entropy has an area behavior with the same leading $\varepsilon$-divergence. Whereas for conformally invariant bulk theories one can use the global conformal equivalence of the wedge with a double cone, there are presently no results for massive theories on null-surfaces of double cones. Such null-surfaces are quite interesting because the geometry in the limit of large double cones is expected to make contact with the classical BondiMetzner-Sachs symmetry. Whereas the quantum symmetries of holography are always defined through their unitaries in the full Hilbert space, the classical BMS symmetry is only defined as an asymptotic transformation.

The difference to previous entropy calculations based on counting eigenstates of the standard time translation Hamiltonian [15] (after modifying the local model by a momentum space cutoff) is that those calculations do not reveal the local origin of the of the area proportionality. There are general reasons which cast serious doubts on global calculations of vacuum entropy and energy [16] which count the contribution from the occupation of globalized energy using the standard time translation Hamiltonian and using a cutoff in momentum space. Such calculations violate the recently formulated principle of local covariance of QFT in curved spacetime [6]. The present calculation does better on this issue, since the Hamiltonian is the same as the one responsible for the Hawking-Unruh effect i.e. adapted to the invariance of the localization region and its horizon. In addition the theory is not modified by momentum space cutoffs; one rather looks at a family of localization entropies for the holographically projected matter with a fuzzy boundary of lightlike extension $\varepsilon$ at the edge of the horizon which is interpreted as an attenuation length. In particular this approach shows that the rather universal behavior of vacuum polarization clouds near boundaries should not to be blamed on the ultraviolet behavior of particular pointlike fields, but is rather a generic physical consequence of the principle of causal localizability. Localized operator algebras are von Neumann factor algebras of hyperfinite type $\mathrm{III}_{1}$; this unique operator algebra (modulo isomorphisms) which peculiar properties (related to localization and thermal aspects) which easily escape the quantum mechanical intuition since the algebras one meets in QM at zero temperature are of type I [17]. Some of the problems black hole physics seems to have with the foundations of QT may find their explanation in these peculiarities.

It has been known for some time that curved spacetimes with a bifurcated Killing horizon (bKh) is analogous to wedges in Minkowski spacetime. A presentation in the setting of operator algebras in curved space time in a form which is most suitable for the calculation of localizationentropy has been given in [9], following prior work in [18][19]. The main difference to the Minkowski situation is that at the beginning one only has a Killing symmetry together with some assumptions about the way it acts geometrically which guaranty the existence of a bKh. As an analog for the vacuum state one needs a state vector $\Omega$ which is invariant under the action of the Killing symmetry. For the Schwarzschild-Kruskal black-hole spacetime this would be the Hartle-

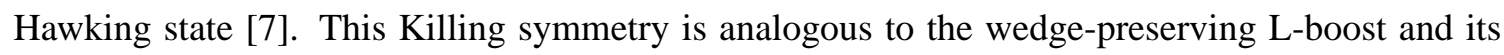
projection onto the $\mathrm{bKh}$ is the candidate for a dilation. It comes as quite as a surprise that it is possible to extend this symmetry to a full Moebius group, including a positive energy lightlike translation [9]. Again the implementing unitary operators are defined on the whole Hilbert space, but their geometric action as a Moebius symmetry is restricted to the horizon. The definition of the net of algebras on the horizon is similar to the Minkowski case in that the wedge regions $W$ 
possess a bKh analog; instead of the Wigner little group (which was used to resolve the transverse locality structure on $\left.\partial W_{a, b}\right)$ one intersects $\mathscr{A}\left(\partial W_{a, b}\right)$ with bulk algebras whose localization region contains the subregion of $\partial W_{a b}$ with a specified transverse extension (tubular neighborhoods). The subtle part of is the construction of the positive energy translation from algebraic properties of the bKh holography. For this and other related details we refer to [9] which is the most authoritative account of this matter. Having arrived at a chiral Moebius symmetry on the horizon, the calculation of localization-entropy is analogous and leads also to the logarithmic divergence in terms of a length scale which is adjusted to dilations.

Equating this entropy density with Bekenstein's classical area density relates the attenuation size $\varepsilon$ of the vacuum polarization cloud to the Planck length. The present purely structural approach does not resolve the question whether different content of bulk matter gives different c-values and which c-values are belonging to which bulk matter. But in case that the holographically projected matter does admit more than one value of $c$ this could lead to a problematization with the matterindependent pure gravity-based Bekenstein density which is usually interpreted as including the contribution coming from quantum matter. In view of the fact that the state of black hole radiation of a collapsing star [20][21] is not really an equilibrium state, the recent operator algebra concept of an entropy flux [22] for stationary states which are not equilibrium states may be more appropriate.

Finally it is important to stress that the notion of holography which is in widespread use in the literature (originally introduced by 't Hooft) is not the same as the one in the present work. The main difference is that the former is thought of as leading to a complete holographic image from which the full bulk theory can be recovered. Such an invertible "duality" relation between bulk and its holographic image, if possible at all, is expected to arise from the still elusive quantum gravity and serves as a postulate whose consequences are to be studied. The present notion of holography onto null-surfaces lacks this uniqueness of inversion. For example it is not possible to reconstruct the localization structure within a wedge $W$ solely from that of its horizon $\partial W$, the reconstruction is limited to those semi-infinite regions which arise as a causal shadow from regions on $\partial W$; the finer substructure e.g. the double cone algebras inside the $W$ bulk are certainly not reconstructible only from subalgebras on $\partial W$. Adding the knowledge about actions of Poincaré transformations outside the 7-parametric subgroup $\mathscr{G}$ of $L F$ (e.g. a lightlike translation moving outside of $L F$ ) the full net of bulk algebras and their pointlike generators may be recovered. Since in the presence of interactions there is no direct local connection between bulk fields and holographic generators, the intervention of operator algebras is an essential aspect of holography of interacting systems. Apart from the structural results used in the present work, the theory of operator algebras as one needs it the physical setting is very much in its infancy and there is presently no good intuitive understanding of how geometric-physical properties in spacetime are related to the positioning of isomorphic copies of the hyperfinite type $\mathrm{III}_{1}$ factor algebra in a common Hilbert space, although it can be shown that any QFT in Minkowski spacetime, including its Poincaré symmetries, can be encoded in this way [23].

The relation of the conjectured quantum gravitational holography on null-surfaces to the one in this paper is similar to that of the conjectured Maldacena [24] to Rehren's algebraic AdS-CFT correspondence [25]. The full invertibility (which justifies the use of the word correspondence) of Rehren's algebraic correspondence results from the exceptional fact that the causal shadows cast by the regions in a conformal brane boundary lead to a complete net of algebras in AdS (related to 
the shared maximal symmetry of bulk and boundary); it has nothing to do with quantum gravity.

\section{References}

[1] P. Jordan, The Present State of Quantum Electrodynamics, in Talks and Discussions of the Theoretical-Physical Conference in Kharkov (May 19.-25., 1929) Physik. Zeitschr. XXX (1929) 700

[2] R. Haag, Local Quantum Physics, Springer-Verlag, Berlin-Heidelberg-New York 1996

[3] M. Duetsch and K. Fredenhagen, Perturbative renormalization and BRST, hep-th/0411196

[4] M. Duetsch and B. Schroer, J.Phys. A33 (2000) 4317, hep-th/9906089

[5] J. Mund, B. Schroer and J. Yngvason, Commun. Math. Phys. 268, (2006) 621, math-ph/0511042

[6] R. Brunetti, K. Fredenhagen and R. Verch, Commun.Math.Phys. 237 (2003) 31, math-ph/0112041

[7] R. M. Wald, General Relativity, the University of Chicago Press 1994

[8] B. Schroer, Class.Quant.Grav. 23 (2006) 5227, hep-th/0507038 and previous work cited therein

[9] D. Guido, R. Longo, J.E. Roberts, R. Verch, Rev.Math.Phys. 13 (2001) 125, math-ph/9906019

[10] R. Brunetti and K. Fredenhagen, Towards a Background Independent Formulation of Perturbative Quantum Gravity, gr-qc/0603079

[11] H.-J. Borchers, D. Buchholz and B. Schroer, Commun. Math. Phys. 219, (2001) 125, hep-th/0003243

[12] G. Lechner, An Existence Proof for Interacting Quantum Field Theories with a Factorizing S-Matrix, math-ph/0601022

[13] W. Driessler, Acta Phys. Austr. 46 (1977) 63

[14] D. Guido, R. Longo and H.-W. Wiesbrock, Commun.Math.Phys. 192 (1998) 217, hep-th/9703129

[15] L. Bombelli, R. K. Kaul, J. Lee and R. Sorkin, Phys. Rev. D 34, (1986) 373

[16] S. Hollands and R. E. Wald, Gen.Rel.Grav. 36 (2004) 2595, gr-qc/040508

[17] J. Yngvason, Rept.Math.Phys. 55 (2005) 135, math-ph/0411058

[18] G. L. Sewell, Ann. Phys. 141 (1982) 201

[19] S. J. Summers and R. Verch, Lett. Math. Phys. 37 (1996) 145

[20] S. W. Hawking, Commun. Math. Phys. 43, (1975) 199

[21] K. Fredenhagen and R. Haag, Commun. Math. Phys. 127 (1990) 1

[22] V. Jakcik and C.-A. Pillet, Comm. Math. Phys. 217 (2001) 285

[23] R. Kaehler and H.-W. Wiesbrock, JMP 42, (2000) 74, and references therein

[24] J. Maldacena, Adv. Theor. Math. Phys. 2 (1998) 231

[25] K.-H. Rehren, Annales Henri Poincare 1 (2000) 607, hep-th/9905179, and QFT Lectures on AdS-CFT, hep-th/0411086 